\title{
Characteristic modes of vibration of Q-balls
}

\author{
Andrei Kovtun ${ }^{1,2, *}$, Emin Nugaev ${ }^{1, * *}$, and Andrey Shkerin ${ }^{3,1, * * *}$ \\ ${ }^{1}$ Institute for Nuclear Research of the Russian Academy of Sciences, 60th October Anniversary prospect \\ 7a, 117312, Moscow, Russia \\ ${ }^{2}$ Moscow Institute of Physics and Technology, Institutskii per. 9, Dolgoprudny, Moscow Region \\ 141700, Russia \\ ${ }^{3}$ Institute of Physics, Ecole Polytechnique Fédérale de Lausanne (EPFL), CH-1015, Lausanne, Switzer- \\ land
}

\begin{abstract}
In this report, based on [1], we study analytically small perturbations of classically stable Q-balls in $3+1$ dimensions. Models with flat and polynomial potentials are considered. We find that large Q-balls in the model with the flat potential possess soft modes. We also find a specific mode for Q-balls near the stability bound, which is related to the decay mode of Q-clouds. For these modes, the perturbation theory is applicable with respect to the relative frequency of an excitation.
\end{abstract}

\section{Introduction}

Q-balls are non-topological solitons arising in complex scalar field theories with the global $U(1)$-invariance $[2,3] .^{1}$ During the years of studies, they found numerous applications in different branches of modern physics. Q-balls are predicted in models of physics beyond the Standard Model [6], and were applied to different problems in astrophysics and cosmology (baryogenesis [7], phase transitions in the Early Universe [8], dark matter [9], alternative to black holes [10]). Beside that, the existence of Q-balls is discussed, e.g., in condensed matter physics [11].

Here we focus on studying spectra of linear fluctuations on top of classically stable Qballs. Analysis of small perturbations of classical solutions is of considerable interest from the theoretical and phenomenological perspectives. Recent examples include the investigation of quasinormal modes of black holes $[12,13]$ or excitations in a non-uniform Bose-Einstein Condensate [14]. The advantage of Q-balls is that they are relatively simple objects and often their analysis can be performed by analytical methods. Below we will use models admitting analytical treatment for both Q-balls and their perturbations. We start in the next section with a brief overview of the main properties of Q-balls. In sections 3 and 4 we study modes of Q-balls in the flat and polynomial potentials. Section 5 contains a summary of our results.

\footnotetext{
*e-mail: andreykpq@gmail.com

**e-mail: emin@ms2.inr.ac.ru

***e-mail: andrey.shkerin@epfl.ch

${ }^{1}$ In case of the local $U(1)$-invariance, one speaks of "gauged Q-balls" [4, 5].
} 


\section{Setup}

Q-balls are localized stationary solutions of classical equations of motion in a theory with the Lagrangian $^{2}$

$$
\mathcal{L}=\left|\partial_{\mu} \phi\right|^{2}-V(|\phi|)
$$

The field configuration of a Q-ball is described by the ansatz

$$
\phi_{0}(x)=f(r) e^{i \omega t}, \quad r=|\vec{x}|,
$$

where $f$ is a monotonically decreasing function of the radial coordinate which obeys the boundary conditions

$$
f^{\prime}(0)=0, \quad f(\infty)=0,
$$

and without loss of generality one can put $\omega>0$.

The existence and stability of Q-balls are due to the presence in the theory of a conserved $U(1)$-charge,

$$
Q=i \int d^{3} x\left(\dot{\phi}_{0}^{*} \phi_{0}-\dot{\phi}_{0} \phi_{0}^{*}\right)=2 \omega \int d^{3} x f^{2} .
$$

Beside the charge, the Q-ball is characterized by its energy,

$$
E=\int d^{3} x\left((\vec{\nabla} f)^{2}-\omega^{2} f^{2}+V(f)\right) .
$$

The existence condition for Q-balls is easily derived, e.g., by the means of the analogy between the equation for the Q-ball profile $f$ and Newton's equation for the particle of unit mass moving in the potential $1 / 2\left(\omega^{2} x^{2}-V(x)\right)$. It reads as follows [3],

$$
\min _{\phi \neq 0} \frac{V(|\phi|)}{|\phi|^{2}} \equiv \omega_{\min }^{2}<\omega^{2}<V^{\prime \prime}(0) \equiv m^{2}
$$

provided that the inequality $\omega_{\min }<m$ holds for the potential $V$.

\section{Perturbations of Q-balls in the flat potential}

In this section, we consider the potential consisting of a parabolic branch followed by a flat branch,

$$
V(|\phi|)=m^{2}|\phi|^{2} \theta\left(1-\frac{|\phi|^{2}}{v^{2}}\right)+m^{2} v^{2} \theta\left(\frac{|\phi|^{2}}{v^{2}}-1\right) .
$$

It is a particular case of the so-called piecewise-parabolic potential [16]. It is known that the theory with this potential allows for analytical investigation of both the Q-balls and linear perturbations above them $[2,17]$. Besides, the potential (7) serves to approximate realistic potentials with the flat direction, which are used, e.g., in describing dark matter by Q-balls [9]. From eq. (6) we see that the mass $m$ of the free particle is the relevant dimensional parameter of the theory. Because of this, below we normalize physical quantities to it, by setting $m=1$.

The properties of Q-balls in the potential (7) are well-known. Depending on the frequency, the solution belongs to one of the three groups, see table 1. Q-balls of the first group have $\omega$ in the interval $0<\omega \leq \omega_{s}$ where $\omega_{s}$ is such that

$$
\left.E\right|_{\omega=\omega_{s}}=\left.m Q\right|_{\omega=\omega_{s}} .
$$

\footnotetext{
${ }^{2}$ It is also possible to consider theories with more than one scalar field; see, e.g., [15].
} 
Table 1: Three groups of Q-balls in the theory (1) with the potential (7). Numerically, $\omega_{s} \approx$ 0.837 and $\omega_{c} \approx 0.960$.

\begin{tabular}{lc}
\hline Q-clouds & $\omega_{c}<\omega<m$ \\
\hline Metastable Q-balls & $\omega_{s}<\omega<\omega_{c}$ \\
\hline Absolutely stable Q-balls & $0<\omega<\omega_{s}$ \\
\hline
\end{tabular}

If $\omega<\omega_{s}$, the energy of the Q-ball is less than the energy of the collection of free particles of the same charge. Hence, the Q-balls of this group are absolutely stable. The second group is characterized by the inequality $\omega_{s}<\omega<\omega_{c}$, where $\omega_{c}$ is the frequency at which

$$
\left.\frac{\partial^{2} E}{\partial Q^{2}}\right|_{\omega=\omega_{c}}=0
$$

The Q-balls with these values of $\omega$ are classically stable, but can decay via tunneling [18]. Finally, the Q-balls with $\omega>\omega_{c}$ are classically unstable. They are also known as Q-clouds [19].

To study small perturbations of Q-balls, we take the ansatz

$$
\phi=\phi_{0}+\chi, \quad \chi(x)=\psi(x) e^{i \omega t},
$$

and substitute it into the equations of motion for $\phi$ and its complex conjugated. Linearizing the result with respect to $\psi$, one obtains

$$
\begin{aligned}
& \left(\partial_{0}+i \omega\right)^{2} \psi-\Delta \psi=-h(r) \psi^{*}-g(r) \psi, \\
& \left(\partial_{0}-i \omega\right)^{2} \psi^{*}-\Delta \psi^{*}=-h(r) \psi-g(r) \psi^{*},
\end{aligned}
$$

where $h$ and $g$ are given by

$$
h(r)=\left.\left(z \frac{d^{2} V}{d z^{2}}\right)\right|_{z=f^{2}(r)}, \quad g(r)=\left.\left(z \frac{d^{2} V}{d z^{2}}+\frac{d V}{d z}\right)\right|_{z=f^{2}(r)}, \quad z=|\phi|^{2} .
$$

In the potential (7) the functions (12) become

$$
h(r)=-\frac{m^{2}}{2} \delta\left(\frac{f(r)}{v}-1\right), \quad g(r)=m^{2} \theta\left(1-\frac{f^{2}(r)}{v^{2}}\right)+h(r) .
$$

We observe that, although in general the equations for $\psi$ and $\psi^{*}$ are not disentangled, in the case of the piece-wise parabolic potential they are separated everywhere expect the single point at which the two branches of the potential are joined. It is this fact that allows to treat analytically both the classical solutions and their linear excitations.

\subsection{Modes of metastable Q-balls and Q-clouds}

Let us first consider the decay mode of Q-clouds. It is described by the spherically-symmetric ansatz of the form [20]

$$
\psi(x)=e^{\tilde{\gamma} t} \zeta(r),
$$

where $\zeta(r)$ is a real function and $\tilde{\gamma}>0$. Substituting eqs. (13) and (14) in eq. (11), one arrives at the linearized equation for $\zeta$. The latter can be solved analytically in the regions $r<R$ and 
$r>R$, where $R$ is the point at which $\left|\phi_{0}\right|=v$. At this matching point, the differential equation on $\zeta$ is reduced to a system of linear equations on the constants entering the general solution. Imposing further the boundary conditions

$$
\zeta^{\prime}(0)=0, \quad \zeta(\infty)=0
$$

determines uniquely both the perturbation profile $\zeta(r)$ and the decay rate $\tilde{\gamma}$, for a fixed frequency of the background Q-ball. ${ }^{3}$ The dependence of $\tilde{\gamma}$ on $\omega$ is shown on the left side of figure 1 . Note also that close to the stability bound

$$
\tilde{\gamma}^{2} \sim \omega-\omega_{c}
$$

We now move to the other side of the stability bound and study the vibrational modes of metastable Q-balls. To this end, one should take the following perturbation ansatz [21],

$$
\psi(x, t)=\left(\psi_{1}^{(l)}(r) e^{i \gamma t}+\psi_{2}^{(l)}(r) e^{-i \gamma t}\right) Y_{l, m}(\theta, \varphi)
$$

where $\gamma$ is real and positive, $\psi_{1}^{(l)}, \psi_{2}^{(l)}$ are real functions, and $Y_{l, m}$ are spherical harmonics. Plugging eq. (17) into eq. (11), one obtains the linearized equations for $\psi_{1,2}^{(l)}$, for any integer $l \geq 0$. Imposing the boundary conditions

$$
\left.\frac{d \psi_{1,2}^{(l)}}{d r}\right|_{r=0}=0, \quad \psi_{1,2}^{(l)}(\infty)=0,
$$

and also

$$
\omega+\gamma<1
$$

which selects the localized "bound states", one arrives at the range of allowable values of $\gamma$, for the fixed $l$ and $\omega$. Interestingly, we find that in the sector of spherically-symmetric modes, $l=0$, there is a unique solution shown on the right side of figure 1 , while for $l>0$ no vibrational modes exist. Close to the critical frequency, one has

$$
\gamma^{2} \sim \omega_{c}-\omega
$$

From eqs. (16) and (20) we see that the vibrational mode of metastable Q-balls and the decay mode of unstable Q-clouds are, in fact, the analytical continuations of each other. It is also important to note that at $\omega \approx \omega_{c}$, the relative oscillation frequency $\gamma$ or the decay rate $\tilde{\gamma}$ can serve as small parameters with respect to which one can build a perturbation theory and obtain the simple expressions for the modes,

$$
\begin{gathered}
\zeta e^{-\tilde{\gamma} t} \sim i f+\tilde{\gamma} \frac{\partial f}{\partial \omega}+O\left(\tilde{\gamma}^{2}\right), \\
\psi_{1} \sim f+\gamma \frac{\partial f}{\partial \omega}+O\left(\gamma^{2}\right), \quad \psi_{2} \sim-f+\gamma \frac{\partial f}{\partial \omega}+O\left(\gamma^{2}\right) .
\end{gathered}
$$

\footnotetext{
${ }^{3}$ It is a general feature of Q-balls that they possess a unique (if any) decay mode [20].
} 

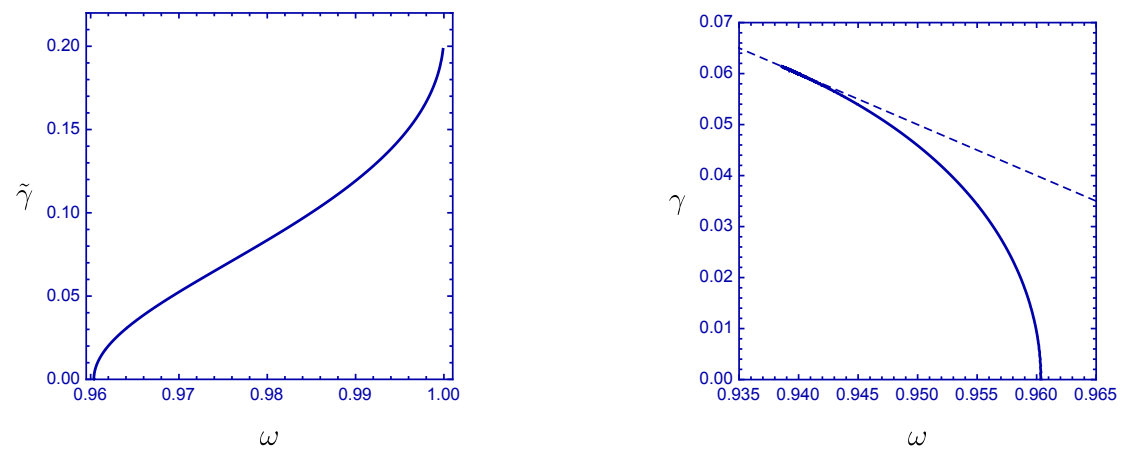

Figure 1: Left: The decay mode of Q-clouds in the theory with the potential (7) with $v=1$. Right: The spherically-symmetric vibrational mode of metastable Q-balls in the potential (7) with $v=1$. The dashed line represents the bound in ineq. (19). The mode ceases to exist at $\omega \approx 0.937$.

\subsection{Modes of absolutely stable Q-balls}

The spectrum of absolutely stable Q-balls is derived in the same way as the vibrational mode of the metastable solutions. The difference is that now there are multiple solutions for any $l \geq 0$. Presented in figure 2 is the family of spherically-symmetric modes. We observe that their number is increased as $\omega \rightarrow 0$. In this limit, the spectrum linearizes and is welldescribed by the equidistant formula

$$
\gamma \simeq \frac{k \omega}{2}, \quad k=1,3,4,5 \ldots
$$

where $k=2$ is excluded since the corresponding solution is singular. When $\omega$ approaches zero, the charge and energy of the Q-ball increase. Hence, large Q-balls in the flat potential possess soft modes in their spectra. The number of the modes is estimated as

$$
N \sim R^{3}
$$

where the matching point $R$ is associated with the size of the soliton. Note finally that the structure of the spectrum with the non-zero orbital momentum is similar to that with $l=0$.

\section{Perturbations of Q-balls in the polynomial potential}

In this section, we consider another featured potential — the one with the power-like asymptotics at large fields. It is known that for a polynomial potential to admit Q-balls, it must be at least of the sixth degree in $|\phi|[3]$. We write it in the following form,

$$
V(|\phi|)=\delta\left(|\phi|^{2}-v^{2}\right)^{2}|\phi|^{2}+\omega_{\min }^{2}|\phi|^{2}
$$

with $\delta>0$, and the minimal frequency $\omega_{\min }$ is related to the free boson mass via

$$
\omega_{\min }=\sqrt{m^{2}-\delta v^{4}} .
$$

The comprehensive analysis of Q-balls in the potential of the sixth degree in 3+1 dimensions was carried out in [22]. Although in this potential the Q-balls cannot be found in an 


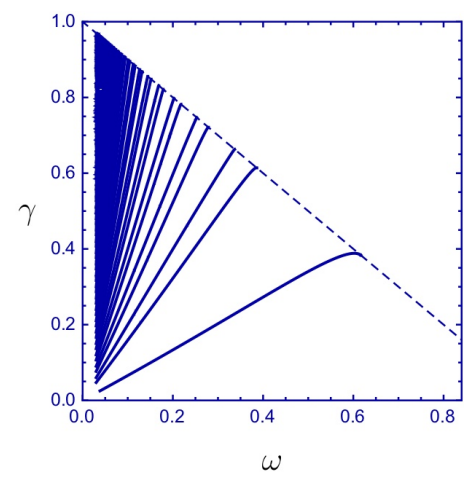

Figure 2: Spherically-symmetric vibrational modes of absolutely stable Q-balls in the potential (7) with $v=1$.

analytic form, the analytical analysis is possible in the thin-wall limit which is achieved close to the lower frequency bound. The accuracy of the thin-wall approximation is controlled by the small parameter

$$
\epsilon=\omega-\omega_{\min } .
$$

The Q-ball profile is approximated by the thin-wall ansatz,

$$
f(r)=f_{0} \theta\left(1-\frac{r}{\rho}\right),
$$

where $\rho$ is the radius of the soliton and $f_{0}$ is the magnitude of the field inside it. To the leading order in $\epsilon$, we have

$$
\begin{aligned}
f_{0} & =v(1+O(\epsilon)), \\
\rho & =\frac{\sqrt{\delta} v^{2}}{2 \omega_{\min } \epsilon}+O(1) .
\end{aligned}
$$

Proceeding as in the case of the flat potential, we take the perturbation asatz (17) for vibrational modes and substitute it into eq. (11), where $g$ and $h$ are now given by

$$
\begin{aligned}
& h(r)=-4 \delta v^{2} f^{2}(r)+6 \delta f^{4}(r), \\
& g(r)=m^{2}-8 \delta v^{2} f^{2}(r)+9 \delta f^{4}(r),
\end{aligned}
$$

and $f(r)$ is determined by eqs. (28), (29). The resulting equations are then solved analytically with the boundary conditions (18) and (19). This gives the spectrum of vibrational modes $\gamma=\gamma(\omega)$ which is plotted in figure 3 for the case $l=0$ and for a particular choice of the parameters in eq. (25). We observe the same properties of the spectrum as in the case of the flat piece-wise potential (7). In particular, the spectrum linearizes in the limit $\omega \rightarrow \omega_{\min }$. In this limit, it is approximated by the formula

$$
\gamma=\frac{2 \omega_{\min }}{m} \mu_{n, l+\frac{1}{2}} \epsilon, \quad n=0,1,2, \ldots
$$

where $\mu_{n, l+\frac{1}{2}}$ is the $\mathrm{n}$-th zero of the $(l+1 / 2)$-order Bessel function of the first kind. Furthermore, the number of modes is given by

$$
N \sim\left(\frac{m-\omega_{\min }}{\epsilon} \frac{m}{\omega_{\min }}\right)^{3} \sim \rho^{3},
$$




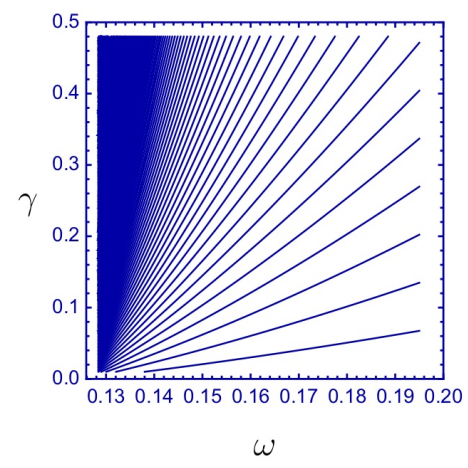

Figure 3: The spectrum of spherically-symmetric perturbations of large Q-balls in the potential (25) with $\delta=1.5, v=0.9$ and $m=1$.

showing again the same counting as for the Q-balls in the flat potential.

\section{Conclusion}

To summarize, in this report we presented the results of the analytical studies of the linear perturbations of Q-balls in theories with the flat and polynomial potentials. We found that in the flat potential the spectra of large Q-balls contain an amount of soft modes. This fact is easily understood in view of the flatness of the potential in the interior of a Q-ball at the scale $\omega^{-1}$. We also showed that at some frequencies the Q-ball may not have bound states described by the ansatz (11). Next, the Q-balls with the near-critical frequencies possess one spherically-symmetric vibrational mode which can be continued analytically to the instability region where it becomes the decay mode of Q-clouds. In the vicinity of the critical point, the perturbation theory can be built with respect to the relative frequency of the oscillation. Finally, it was found that the total amount of modes is proportional to the internal volume of a Q-ball, at least for large enough solitons. This result is to be expected in view of the close, although not exact, analogy between the localized modes of a soliton composed of the complex scalar field and bound states in the potential well, described by the Schrodinger equation.

\section{Acknowledgments}

This work was supported by the Russian Science Foundation grant RSF 16-12-10-494.

\section{References}

[1] A. Kovtun, E. Nugaev, A. Shkerin (2018), 1805.03518

[2] G. Rosen, J.Math.Phys. 9, 996 (1968)

[3] S.R. Coleman, Nucl. Phys. B262, 263 (1985), [Erratum: Nucl. Phys.B269,744(1986)]

[4] K.M. Lee, J.A. Stein-Schabes, R. Watkins, L.M. Widrow, Phys. Rev. D39, 1665 (1989)

[5] I.E. Gulamov, E.Ya. Nugaev, M.N. Smolyakov, Phys. Rev. D89, 085006 (2014), 1311.0325

[6] A. Kusenko, Phys. Lett. B405, 108 (1997), hep-ph/9704273 
[7] I. Affleck, M. Dine, Nucl. Phys. B249, 361 (1985)

[8] J.A. Frieman, G.B. Gelmini, M. Gleiser, E.W. Kolb, Phys. Rev. Lett. 60, 2101 (1988)

[9] A. Kusenko, M.E. Shaposhnikov, Phys. Lett. B418, 46 (1998), hep-ph/9709492

[10] S. Troitsky, JCAP 1611, 027 (2016), 1510.07132

[11] Yu.M. Bunkov, G.E. Volovik, Phys. Rev. Lett. 98, 265302 (2007), cond-mat/0703183

[12] R. Konoplya, A. Zhidenko, Phys. Lett. B756, 350 (2016), 1602 . 04738

[13] V. Cardoso, S. Hopper, C.F.B. Macedo, C. Palenzuela, P. Pani, Phys. Rev. D94, 084031 (2016), 1608.08637

[14] F. Dalfovo, S. Giorgini, L.P. Pitaevskii, S. Stringari, Rev. Mod. Phys. 71, 463 (1999)

[15] R. Friedberg, T.D. Lee, A. Sirlin, Phys. Rev. D13, 2739 (1976)

[16] S. Theodorakis, Phys. Rev. D61, 047701 (2000)

[17] I.E. Gulamov, E.Ya. Nugaev, M.N. Smolyakov, Phys. Rev. D87, 085043 (2013), 1303.1173

[18] D. Levkov, E. Nugaev, A. Popescu, JHEP 12, 131 (2017), 1711.05279

[19] M.G. Alford, Nucl. Phys. B298, 323 (1988)

[20] A.G. Panin, M.N. Smolyakov, Phys. Rev. D95, 065006 (2017), 1612.00737

[21] M.N. Smolyakov, Phys. Rev. D97, 045011 (2018), 1711.05730

[22] M. Mai, P. Schweitzer, Phys. Rev. D86, 076001 (2012), 1206. 2632 